\title{
A Shared Cancer Follow-Up Model of Care Between General Practitioners and Radiation Oncologists for Patients With Breast, Prostate, and Colorectal Cancer: Protocol for a Mixed Methods Implementation Study
}

Tiffany Sandell ${ }^{1,2}$, BA, MPH, MSc; Heike Schütze ${ }^{2,3}$, BSc, MPH, PhD; Andrew Miller ${ }^{1,2}$, BMed, BSc, Grad Dip, MInfCommTech, FRANZCR, FAIDH

\footnotetext{
${ }^{1}$ Wollongong Hospital, Wollongong, Australia

${ }^{2}$ University of Wollongong, Wollongong, Australia

${ }^{3}$ University of New South Wales, Sydney, Australia
}

\section{Corresponding Author:}

Tiffany Sandell, BA, MPH, MSc

Wollongong Hospital

Loftus Street

Wollongong, 2500

Australia

Phone: 6124222500

Email: tiffany.sandell@health.nsw.gov.au

\begin{abstract}
Background: The rising incidence of cancer and increasing numbers of cancer survivors have resulted in the need to find alternative models of care for cancer follow-up care. The acceptability for follow-up care in general practice is growing, and acceptance increases with shared-care models where oncologists continue to oversee the care. However, a major barrier to this model is the effective exchange of information in real time between oncologists and general practitioners. Improved communication technology plays an important role in the acceptability and feasibility of shared cancer follow-up care.

Objective: The aim of this study is to evaluate the feasibility and acceptability of a shared cancer follow-up model of care between patients, general practitioners and radiation oncologists.

Methods: This is a mixed methods, multisite implementation study exploring shared follow-up care for breast, colorectal, and prostate cancer patients treated with curative radiotherapy in New South Wales, Australia. This study uses web-based technology to support general practitioners in performing some aspects of routine radiotherapy follow-up care, while being overseen by a radiation oncologist in real time. The study has two phases: Phase 1 is designed to establish the level of agreement between general practitioners and radiation oncologists and Phase 2 is designed to implement shared follow-up care into practice and to evaluate this implementation.
\end{abstract}

Results: Recruitment of radiation oncologists, patients, and general practitioners commenced in December 2020 and will continue until February 2021. Data collection will occur during 2021, and data will be ready for analysis by the end of 2021.

Conclusions: Few studies have investigated the role of health technologies in supporting communication deficiencies for shared cancer follow-up care. The implementation and evaluation of models of care need to be conducted using a person-centered approach that is responsive to patients' preferences and needs. Should the findings of the study be acceptable and feasible to radiation oncologists, general practitioners, and patients, it can be quickly implemented and expanded to other tumor groups or to medical oncology and hematology.

Trial Registration: Australian New Zealand Clinical Trials Registry ACTRN12620001083987; http://www.anzctr.org.au/Trial/Registration/TrialReview.aspx?id=380057

International Registered Report Identifier (IRRID): PRR1-10.2196/21752

(JMIR Res Protoc 2021;10(1):e21752) doi: 10.2196/21752 


\section{KEYWORDS}

radiation oncology; general practice; health technology; communication; cancer; shared care; follow-up

\section{Introduction}

The increasing incidence of cancer, coupled with improved survivorship, has resulted in higher demand for cancer follow-up care [1-3]. This has led to the sustainability of oncologist-led cancer follow-up care in the secondary health setting being questioned $[4,5]$ and to a call for alternative models of cancer follow-up care [6,7]. There is a growing body of literature on the benefits of shared cancer follow-up models between general practitioners and oncologists [8]; however, this is yet to be integrated into routine practice.

Randomized controlled trials have shown that cancer follow-up care delivered by a general practitioner in the primary health care setting produces no difference in the rate of recurrence or quality of life compared to cancer follow-up with an oncologist [9-11]. General practitioners are willing to take a greater role in cancer follow-up care [12] provided they are supported by the oncologist [13-16] and the oncologist maintains overall responsibility [17].

Despite an acceptance by patients for their general practitioner to be involved in their follow-up care, barriers to shared care exist. The barriers are role clarification [18-20] and effective two-way communication [21-25]. There is a need for a robust information-sharing system that allows both the general practitioner and the overseeing oncologist to be involved in the follow-up care. Real-time and open access to patient information is crucial to coordinate the care of cancer survivors appropriately [26-28].

At present, cancer patients maintain follow-up with their oncologists in the secondary health care setting, and routine communication is transferred from the oncologist to the general practitioner via letter or secure email. In the case where a general practitioner has undertaken a cancer-specific follow-up, it is uncommon for the general practitioner to communicate their findings to the oncologist. This study will trial a web-based technology to breach the communication divide between the general practitioner and the oncologist so that they can work together collaboratively, should patients choose a shared-care model.

To our knowledge, there is currently no system that supports the involvement of general practitioners in shared cancer follow-up care where the radiation oncologist can oversee the care. This study trials a web-based system that allows general practitioners to undertake routine aspects of cancer follow-up care, while sharing the data with oncologists at the hospital in real time so that they can continue to monitor, oversee, and maintain responsibility for the patient.

This research aims to evaluate the feasibility and acceptability of a shared cancer follow-up model of care between patients, general practitioners and radiation oncologists. The objectives of this study are to implement a model of care using a web-based system that transfers clinical information between the general practitioner and radiation oncologist in real time, to determine the level of agreement between general practitioners and oncologists completing a standardized follow-up assessment, and to establish the feasibility and acceptability of this model of care.

\section{Methods}

\section{Study Design}

This research is a mixed methods, multisite implementation study for breast, colorectal, and prostate cancer patients who have undertaken curative radiotherapy treatment. Mixed methods investigations involve integrating quantitative and qualitative data collection and analysis into a single study [29] and can strengthen the credibility of evidence and evaluation [30].

The study will implement the shared cancer follow-up model of care into practice at baseline (Phase 1) and at 6 months postrecruitment (Phase 2) (see Figure 1). During Phase 1, there will be a standard clinical review by the radiation oncologist as per the patient's routine follow-up schedule, plus an additional follow-up review by the general practitioner using the same standardized follow-up assessment. This first phase will determine the level of agreement between general practitioners and radiation oncologists when completing the same radiotherapy follow-up clinical assessment on the patient. This first phase is essential, as it informs the educational and training requirements for general practitioners. By demonstrating the level of agreement, it reassures both the general practitioner and radiation oncologist that the general practitioner can reliably conduct a cancer-specific follow-up review. 
Figure 1. Flow diagram of study phases. GP: general practitioner.

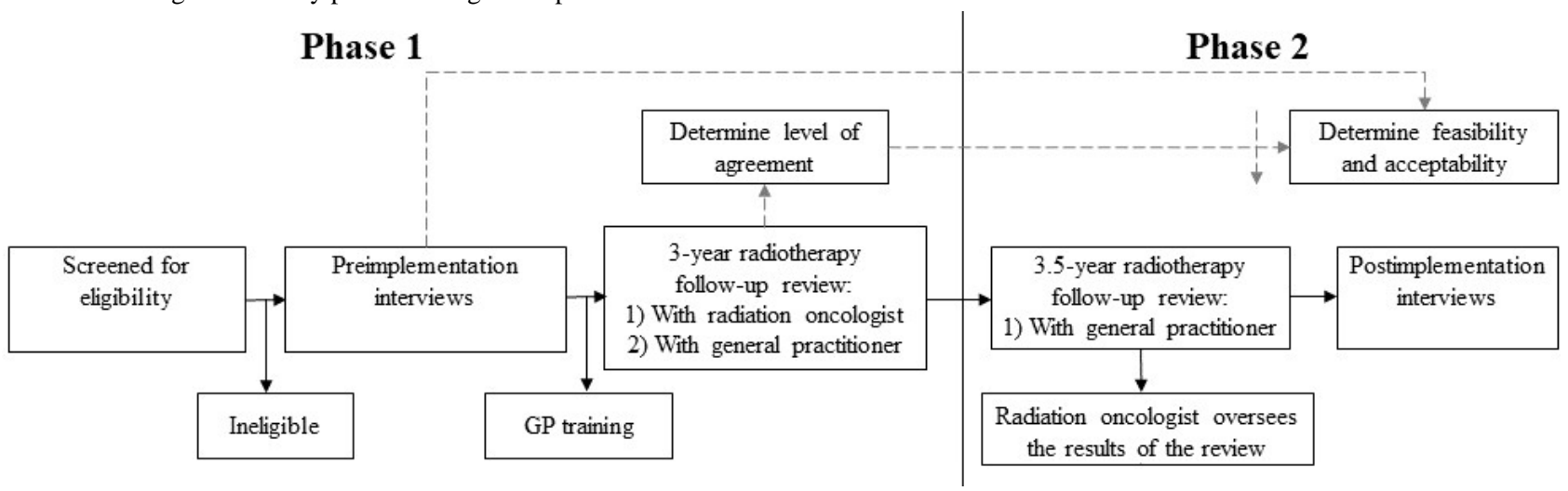

The second phase of the study is the implementation of the shared cancer follow-up model of care into practice. The patient will visit their general practitioner at 3.5 years follow-up for a radiation oncology-specific follow-up appointment. The results will be transferred to the hospital, and the patient's radiation oncologist will be alerted by an automatic quality checklist to review the outcomes of the review in real time on the hospital's oncology information system. The system has a rapid referral built into it in the case of adverse events or should the general practitioner suspect cancer recurrence.

\section{Study Setting}

The research will be conducted within the Illawarra Shoalhaven Local Health District (ISLHD) region in New South Wales, Australia. The ISLHD provides public health services to over 400,000 people and cancer services to almost 9000 people annually (ie, medical oncology, hematology, and radiation oncology). Radiation oncology outpatient services are provided at Wollongong Hospital (ie, tertiary hospital and regional care) and the Shoalhaven District Memorial Hospital (ie, secondary hospital and rural care).

The ISLHD radiation oncology service consults and treats over 1400 patients with radiotherapy and conducts over 5000 follow-up consultations annually. The service has experienced a $20 \%$ increase in follow-up consultations over a 5-year period (2015-2019), and treatment activity is projected to increase by $18 \%$ by 2031 . A substantial proportion of radiotherapy treatment at each site is attributed to breast, colorectal, and prostate cancer.

The study will take place at the two hospital radiation oncology outpatient clinics and in the referring general practices. The relationship between general practice and local health districts in Australia is increasingly pivotal to the health system. General practice in Australia is typically comprised of small businesses with an average of three to five general practitioners, and a universal medical insurance scheme (ie, Medicare) covers all or part of a person's cost to visit a general practitioner [31].

\section{Local Follow-Up Guidelines}

While there are many statements regarding "standard follow-up practices," postradiotherapy follow-up for patients varies greatly depending on the disease type, the oncologist's preference, and the patient's preference. At the ISLHD, a visit 6 weeks after radiotherapy is routine for most cases to review the settling of acute side effects. The pattern of remaining follow-up sessions for all cancers will include a period of every 3 months for the first year and every 6 months for the second year, followed by yearly reviews and then, finally, discharge from follow-up. For many cancers, a 5-year period of follow-up is common.

At the ISLHD, an acceptable practice for breast cancer patients' postradiotherapy follow-up care would be a follow-up at 6 weeks, then every 3 months for 2 years, then every 6 months to 5 years, and then yearly to 10 years. An acceptable practice for colorectal cancer patients would be follow-ups every 6 months for the first year and then yearly to 5 years. An acceptable practice for prostate cancer patients would be follow-ups every 6 months or yearly to 5 years. However, the actual frequency depends on the individual patient's health, stage, and treatment and their preference for whom to see; in addition, there is currently no early discharge, transfer of care, or shared care for radiation oncology follow-up care to general practitioners.

\section{Health Technology}

The free and open source software framework PROsaiq (Didymo Pty Ltd) will be used [32]. PROsaiq is based around a web server that extracts assessments from inside the oncology information system and encodes the assessment data into XForms (ie, an XML format used for collecting inputs from web forms), which is then presented as a webpage in a web browser. When the clinical assessment is completed on a smart device (ie, phone, computer, or tablet), the clinical assessment is returned to the web server and converted into a Health Level Seven (HL7) message; HL7 is an accepted international communication standard for clinical systems, such as those comprising laboratory information. The HL7 message is presented to the oncology information system MOSAIQ (Elekta $\mathrm{AB})$, where it is imported to become part of the patient's oncological record.

Australia is equipped with reliable internet capability, and the webpage link will be made available to the general practitioner by integrating it into a current local system that they utilize. The general practitioner will complete the patient follow-up clinical assessments using PROsaiq, and the radiation oncologist will receive an automated alert in real time to review the results at the hospital. PROsaiq has been trialed for the collection of cancer patient-reported quality-of-life outcomes from patients and has demonstrated its operational feasibility [33]. 


\section{Eligibility Criteria}

To be eligible for the study, patients must (1) have a previous diagnosis of colorectal, breast, or prostate cancer; (2) have completed curative-intent radiotherapy treatment and are due for their 3-year review; (3) be over 18 years of age; (4) be able to understand and speak English; and (5) have a general practitioner willing to participate. Patients who do not meet these criteria will be excluded, as will patients who have suspected or confirmed recurrence of cancer.

Patients 3 years posttreatment have been selected, as it was deemed a safe time period by the oncologists for a feasibility study, and the patients will have experienced the standard oncologist-led follow-up model. Participants can withdraw at any stage up until data analysis.

\section{Sample Size}

The sample will consist of 20 triads comprising the patient, their radiation oncologist, and their general practitioner, for a total of 35 to 45 participants. A total of 10 patients will be from the Wollongong Cancer Centre (ie, regional) and 10 will be from the Shoalhaven Cancer Centre (ie, rural).

Sample size guidelines for qualitative interviews suggest that a range between 20 and 30 interviews is adequate for each group to reach data saturation [34]. The sample size for the quantitative level of agreement data requires a minimum of 5 samples; however, to increase the confidence interval, a higher sample is required [35].

\section{Recruitment}

The radiation oncologists will review their follow-up clinic lists from both sites and screen for initial inclusion criteria. The researcher will invite each patient to participate via a postal letter on behalf of the radiation oncologist. Once each patient consents to participate, their general practitioner will be invited. General practitioners will be eligible for continuing professional development points for participating. If the general practitioners do not consent to participate, the patient will not be eligible.

\section{Implementation}

The foundation of this shared cancer follow-up model of care is that clinician communication exchange is two-way and in real time, while the radiation oncologist continues to oversee the follow-up care. The model includes real-time transfer of results, internal system alerts, and rapid referral to address any issues that may arise. During this study, patients maintain their current specialist standard follow-up care, with all relevant specialists, and will continue follow-up care with their radiation oncologist upon completion of the study.

General practitioners will complete a standardized online radiation oncology course developed by the Cancer Institute New South Wales [36]. The course developed for health professionals addresses the principles of radiation therapy, patient assessment grading systems of side effects, and supportive care management. General practitioners will receive one-on-one training by a radiation oncologist that includes localized radiotherapy-specific follow-up care, a review of the recruited patient's treatment background, and a demonstration of the clinical follow-up assessment that the general practitioner will use in the patient's follow-up review.

\section{Data Collection}

\section{Overview}

The PROsaiq software will be used to administer clinical assessments. The assessments were compiled internally at the ISLHD for follow-up of radiotherapy patients. These clinical assessments review physical items on a scale from 0 to 4 for items specific to radiotherapy follow-up care, such as pain, fatigue, physical performance, bowel issues, urinary issues, and appetite (see Table 1). The included scales were sourced from the Radiation Therapy Oncology Group scales [37] and the Common Terminology Criteria for Adverse Events, version 3.0 [38].

Table 1. Radiation oncology follow-up standardized clinical assessment.

\begin{tabular}{ll}
\hline Tumor type & Clinical assessments \\
\hline Breast & $\begin{array}{l}\text { Fatigue, ECOG (Eastern Cooperative Oncology Group) Performance Status, appetite, weight loss, chest and breast pain, telangiectasia, } \\
\text { lymphedema-related fibrosis, and disease state (ie, local, regional, or distant) }\end{array}$ \\
Colorectal & Fatigue, ECOG Performance Status, appetite, weight loss, proctitis, pelvic pain, vomiting, and diarrhea \\
Prostate & Fatigue, ECOG Performance Status, erectile dysfunction, dysuria, and rectal hemorrhage \\
\hline
\end{tabular}

\section{Quantitative Data}

The quantitative data will be collected from Phase 1. The radiation oncologist will enter the clinical assessment directly into the oncology information system, while the general practitioners will enter the clinical assessment on the webpage link that will be provided to the general practitioner. Both sets of data from these clinical assessments will be stored in the hospital oncology information system.

\section{Qualitative Data}

At pre- and postimplementation, participants (ie, patients, general practitioners, and radiation oncologists) will participate in semistructured interviews following a topic guide about radiotherapy follow-up care and their experience of shared care. Demographic data will be collected for all participants (ie, age, sex, level of education, and working years). The interviews will be audio-recorded and transcribed verbatim in preparation for thematic analysis in NVivo (QSR International). 


\section{Data Analyses}

\section{Quantitative Data}

The clinical assessment data will be extracted from the oncology information system; the Cohen $\kappa$ value and percent agreement for each variable from Table 1 will determine the level of agreement between general practitioners and radiation oncologists. The agreement will assess the concordance between two measurements of each variable with the expectation that there will be near-perfect agreement on each item $(>0.81)$. The results of the analysis and level of agreement will be presented to the general practitioners and radiation oncologists to guide any additional education and training.

\section{Qualitative Data}

Thematic analysis is a commonly used analytical approach for qualitative data in implementation studies [39]. This involves mapping the transcribed data and emergent themes onto a priori domains. The themes will be compared across the regional and rural sites (ie, Wollongong and Shoalhaven) and triangulated between radiation oncologists, patients, and general practitioners.

\section{Ethics Approval and Trial Registration}

Ethics approval was received on May 12, 2020, from the Joint University of Wollongong and the ISLHD Human Research Ethics Committee (2020/ETH00301). The trial was registered with the Australian New Zealand Clinical Trials Registry on October 20, 2020 (ACTRN12620001083987).

\section{Results}

Recruitment of radiation oncologists, patients, and general practitioners commenced in December 2020 and will continue until February 2021. Data collection will occur during 2021, and data will be ready for analysis by the end of 2021 .

\section{Discussion}

\section{Overview}

The important skill set and experience that oncologists have is undisputed. However, there appear to be limited alternate models of cancer follow-up care that address the principles of equity in access, connecting health services, and where the cancer survivor can make an informed decision about their cancer follow-up care. Cancer survivors are more likely to accept shared cancer follow-up care with a general practitioner if their care is overseen by their oncologist [15]. However, effective two-way communication between oncologists and general practitioners is lacking. Improved communication is the strongest enabler to routine shared cancer follow-up care and is an area that is still being established [22,40-42].

Few studies have investigated the role of health technologies in supporting communication deficiencies for shared cancer follow-up care [43]. There have been no explicit recommendations of what type of health technology to use or how to use it. Health technology has been embraced for the collection of patient-reported outcomes of cancer patients during follow-up care, which utilizes the internet to complete online assessments that connect to the hospitals' patient medical files [44]. To our knowledge, using this type of technology between general practitioners and the oncologists is the first of its kind.

The body of literature on the benefits of general practitioner-led and shared cancer follow-up models of care is growing. Although shared follow-up care may not be desired or appropriate for everyone, Australia's oncologist-led model currently leaves limited patient choice as to when, where, and by whom their follow-up care is delivered. A well-informed patient can actively participate in the decision-making process about their care based on their personal circumstances, beliefs, and priorities.

Oncologists, general practitioners, and patients are supportive of a model of shared care [15,16,45]; however, any model developed needs to address the two-way communication barrier and be evaluated for acceptability [46]. The outcomes of this study may lead to a longitudinal implementation to measure patient satisfaction, cost-benefit analysis, health economic analysis, management of rapid referrals, and long-term outcomes of patients.

\section{Limitations}

Possible limitations of this research are the number of participants needed to determine the level of agreement; the research team will monitor this. Another limitation identified is that the general practitioners and radiation oncologists recruited may assess the same oncological patients from a different viewpoint due to differences in training. The researcher will assist in the coordination of appointments and try to minimize the impact on the patients and health professionals.

\section{Acknowledgments}

We would like to thank the Radiation Oncology Department and Research Central at the ISLHD. This research is supported by an Australian Government Research Training Program scholarship.

\section{Conflicts of Interest}

None declared.

\section{References}

1. Adam R, Watson E. The role of primary care in supporting patients living with and beyond cancer. Curr Opin Support Palliat Care 2018;12(3):261-267. [doi: 10.1097/spc.0000000000000369] 
2. Bray F, Ferlay J, Soerjomataram I, Siegel R, Torre L, Jemal A. Global cancer statistics 2018: GLOBOCAN estimates of incidence and mortality worldwide for 36 cancers in 185 countries. CA Cancer J Clin 2018 Nov;68(6):394-424 [FREE Full text] [doi: $10.3322 /$ caac. 21492] [Medline: $\underline{30207593}$ ]

3. Torre L, Bray F, Siegel R, Ferlay J, Lortet-Tieulent J, Jemal A. Global cancer statistics, 2012. CA Cancer J Clin 2015 Mar;65(2):87-108 [FREE Full text] [doi: 10.3322/caac.21262] [Medline: 25651787]

4. Berendsen A, Roorda C, Jansen L, de Bock G. Patients' beliefs about the aims of breast cancer follow-up: A qualitative study. Maturitas 2016 Sep;91:140-144. [doi: 10.1016/j.maturitas.2016.06.014] [Medline: 27451332]

5. Roorda C, de Bock G, Scholing C, van der Meer K, Berger M, de Fouw M, et al. Patients' preferences for post-treatment breast cancer follow-up in primary care vs secondary care: A qualitative study. Health Expect 2015 Dec;18(6):2192-2201 [FREE Full text] [doi: 10.1111/hex.12189] [Medline: 24661322]

6. Institute of Medicine. In: Levit L, Balogh E, Nass S, Ganz P, editors. Delivering High-Quality Cancer Care: Charting a New Course for a System in Crisis. Washington, DC: The National Academies Press; 2013.

7. Cancer Australia. Cancer Australia Strategic Plan 2014-2019. Surry Hills, Australia: Cancer Australia; 2014. URL: https:/ /www.canceraustralia.gov.au/sites/default/files/publications/cancer-australias-strategic-plan-2014-2019/pdf/ 2014 strategic plan.pdf [accessed 2021-01-11]

8. Grunfeld E. Cancer survivorship: A challenge for primary care physicians. Br J Gen Pract 2005 Oct;55(519):741-742 [FREE Full text] [Medline: 16212847]

9. Grunfeld E, Julian J, Pond G, Maunsell E, Coyle D, Folkes A, et al. Evaluating survivorship care plans: Results of a randomized, clinical trial of patients with breast cancer. J Clin Oncol 2011 Dec 20;29(36):4755-4762. [doi: 10.1200/JCO.2011.36.8373] [Medline: 22042959]

10. Johnson C, Saunders C, Phillips M, Emery J, Nowak A, Overheu K, et al. Randomized controlled trial of shared care for patients with cancer involving general practitioners and cancer specialists. J Oncol Pract 2015 Sep;11(5):349-355 [FREE Full text] [doi: 10.1200/jop.2014.001569]

11. Grunfeld E, Levine M, Julian J, Coyle D, Szechtman B, Mirsky D, et al. Randomized trial of long-term follow-up for early-stage breast cancer: A comparison of family physician versus specialist care. J Clin Oncol 2006 Feb 20;24(6):848-855. [doi: $\underline{10.1200 / J C O .2005 .03 .2235}$ ] [Medline: $\underline{16418496]}$

12. Fidjeland H, Brekke M, Vistad I. General practitioners' attitudes toward follow-up after cancer treatment: A cross-sectional questionnaire study. Scand J Prim Health Care 2015;33(4):223-232 [FREE Full text] [doi: 10.3109/02813432.2015.1118836] [Medline: 26649452]

13. Lang V, Walter S, Fessler J, Koester M, Ruetters D, Huebner J. The role of the general practitioner in cancer care: A survey of the patients' perspective. J Cancer Res Clin Oncol 2017 May;143(5):895-904. [doi: 10.1007/s00432-017-2343-4] [Medline: 28188361]

14. Laporte C, Vaure J, Bottet A, Eschalier B, Raineau C, Pezet D, et al. French women's representations and experiences of the post-treatment management of breast cancer and their perception of the general practitioner's role in follow-up care: A qualitative study. Health Expect 2017 Aug;20(4):788-796 [FREE Full text] [doi: 10.1111/hex.12518] [Medline: 27899006]

15. Schütze H, Chin M, Weller D, Harris M. Patient, general practitioner and oncologist views regarding long-term cancer shared care. Fam Pract 2018 May 23;35(3):323-329 [FREE Full text] [doi: 10.1093/fampra/cmx105] [Medline: 29045632]

16. Hall S, Samuel L, Murchie P. Toward shared care for people with cancer: Developing the model with patients and GPs. Fam Pract 2011 Oct;28(5):554-564. [doi: 10.1093/fampra/cmr012] [Medline: 21467132]

17. Cheung W. Sharing clinical responsibility in personalized management of colorectal cancer. Oncol Exch 2014 Feb;13(1):11-12 [FREE Full text]

18. Nyarko E, Metz J, Nguyen G, Hampshire M, Jacobs L, Mao J. Cancer survivors' perspectives on delivery of survivorship care by primary care physicians: An internet-based survey. BMC Fam Pract 2015 Oct 20;16:143 [FREE Full text] [doi: 10.1186/s12875-015-0367-x] [Medline: 26486311]

19. Potosky A, Han P, Rowland J, Klabunde C, Smith T, Aziz N, et al. Differences between primary care physicians' and oncologists' knowledge, attitudes and practices regarding the care of cancer survivors. J Gen Intern Med 2011 Dec;26(12):1403-1410 [FREE Full text] [doi: 10.1007/s11606-011-1808-4] [Medline: 21785923]

20. Sisler J, Chaput G, Sussman J, Ozokwelu E. Follow-up after treatment for breast cancer: Practical guide to survivorship care for family physicians. Can Fam Physician 2016 Oct;62(10):805-811 [FREE Full text] [Medline: 27737976]

21. Dahlhaus A, Vanneman N, Guethlin C, Behrend J, Siebenhofer A. German general practitioners' views on their involvement and role in cancer care: A qualitative study. Fam Pract 2014 Apr;31(2):209-214. [doi: 10.1093/fampra/cmt088] [Medline: 24465025]

22. Dicicco-Bloom B, Cunningham R. The experience of information sharing among primary care clinicians with cancer survivors and their oncologists. J Cancer Surviv 2013 Mar;7(1):124-130. [doi: 10.1007/s11764-012-0253-7] [Medline: 23184488]

23. Franco K, Shuk E, Philip E, Blanch-Hartigan D, Parker P, Matasar M, et al. Communication between oncologists and lymphoma survivors during follow-up consultations: A qualitative analysis. J Psychosoc Oncol 2017;35(5):513-530 [FREE Full text] [doi: 10.1080/07347332.2017.1313352] [Medline: 28358241] 
24. Lawn S, Fallon-Ferguson J, Koczwara B. Shared care involving cancer specialists and primary care providers - What do cancer survivors want? Health Expect 2017 Oct;20(5):1081-1087 [FREE Full text] [doi: 10.1111/hex.12551] [Medline: $\underline{28467626}$

25. Lizama N, Johnson C, Ghosh M, Garg N, Emery J, Saunders C. Keeping primary care. Asia Pac J Clin Oncol 2015 Jun;11(2):152-159. [doi: 10.1111/ajco.12327] [Medline: 25560434]

26. Braun T, Hagen N, Smith C, Summers N. Oncologists and family physicians. Using a standardized letter to improve communication. Can Fam Physician 2003 Jul;49:882-886 [FREE Full text] [Medline: 12901484]

27. O'Brien M, Grunfeld E, Sussman J, Porter G, Mobilio M. Views of family physicians about survivorship care plans to provide breast cancer follow-up care: Exploration of results from a randomized controlled trial. Curr Oncol 2015 Aug;22(4):252-259 [FREE Full text] [doi: 10.3747/co.22.2368] [Medline: 26300663]

28. Barnes E, Chow E, Danjoux C, Tsao M. Collaboration between primary care physicians and radiation oncologists. Ann Palliat Med 2017 Jan;6(1):81-86 [FREE Full text] [doi: 10.21037/apm.2016.11.03] [Medline: 28209071]

29. Tashakkori A, Teddlie C, editors. SAGE Handbook of Mixed Methods in Social \& Behavioral Research. 2nd edition. Thousand Oaks, CA: SAGE Publications; 2010.

30. Patton MQ. Qualitative Research \& Evaluation Methods: Integrating Theory and Practice. 4th edition. Thousand Oaks, CA: SAGE Publications; 2015.

31. Britt H, Miller G, Bayram C, Henderson J, Valenti L, Harrison C, et al. A Decade of Australian General Practice Activity 2006-07 to 2015-16. General Practice Series No. 41. Sydney, Australia: Sydney University Press; 2016. URL: https://tinyurl. com/yyaeyrjs [accessed 2021-01-11]

32. Schuler T, Miller AA. PROsaiq: A smart device-based and EMR-integrated system for patient-reported outcome measurement in routine cancer care. J Radiat Oncol Inform 2014;6(1):111-131 [FREE Full text] [doi: 10.5166/jroi-6-1-24]

33. Girgis A, Durcinoska I, Arnold A, Kaadan N, Miller A, Descallar J, et al. Phase III non-randomized controlled trial of PROMPT-Care, an eHealth intervention utilizing patient reported outcomes in routine clinical care: Impact on emergency department presentations. J Clin Oncol 2019 May 20;37(15_suppl):6510. [doi: 10.1200/jco.2019.37.15 suppl.6510]

34. Creswell JW. Qualitative Inquiry and Research Design: Choosing Among Five Approaches. Thousand Oaks, CA: SAGE Publications; 1998.

35. McHugh ML. Interrater reliability: The kappa statistic. Biochem Med (Zagreb) 2012;22(3):276-282 [FREE Full text] [Medline: 23092060]

36. Radiation oncology. eviQ Education. St Leonards, Australia: Cancer Insititute NSW; 2020. URL: https://education.eviq.org.au/ courses/radiation-oncology/radiation-oncology [accessed 2020-10-27]

37. Cox JD, Stetz J, Pajak T. Toxicity criteria of the Radiation Therapy Oncology Group (RTOG) and the European Organization for Research and Treatment of Cancer (EORTC). Int J Radiat Oncol Biol Phys 1995 Mar 30;31(5):1341-1346. [doi: 10.1016/0360-3016(95)00060-C] [Medline: 7713792]

38. Trotti A, Colevas A, Setser A, Rusch V, Jaques D, Budach V, et al. CTCAE v3.0: Development of a comprehensive grading system for the adverse effects of cancer treatment. Semin Radiat Oncol 2003 Jul;13(3):176-181. [doi: 10.1016/S1053-4296(03)00031-6] [Medline: 12903007]

39. Nowell L, Norris J, White D, Moules N. Thematic analysis. Int J Qual Methods 2017 Oct 02;16(1):1-13 [FREE Full text] [doi: $10.1177 / 1609406917733847]$

40. Sakorafas GH, Tsiotou AG, Pavlakis G. Follow-up after primary treatment for breast cancer. Acta Oncol 2000;39(8):935-940. [doi: 10.1080/02841860050215918] [Medline: 11207000]

41. Allam O, Gray A, Bailey H, Morrey D. Primary care oncology: Addressing the challenges. Inform Prim Care 2006;14(3):167-173 [FREE Full text] [doi: 10.14236/jhi.v14i3.627] [Medline: 17288702]

42. Aubin M, Vézina L, Verreault R, Fillion L, Hudon E, Lehmann F, et al. Family physician involvement in cancer care follow-up: The experience of a cohort of patients with lung cancer. Ann Fam Med 2010;8(6):526-532 [FREE Full text] [doi: 10.1370/afm.1171] [Medline: 21060123]

43. Dickinson R, Hall S, Sinclair J, Bond C, Murchie P. Using technology to deliver cancer follow-up: A systematic review. BMC Cancer 2014 May 03;14:311 [FREE Full text] [doi: 10.1186/1471-2407-14-311] [Medline: 24885758]

44. Girgis A, Durcinoska I, Levesque J, Gerges M, Sandell T, Arnold A, PROMPT-Care Program Group. eHealth system for collecting and utilizing Patient Reported Outcome Measures for Personalized Treatment and Care (PROMPT-Care) among cancer patients: Mixed methods approach to evaluate feasibility and acceptability. J Med Internet Res 2017 Oct 02;19(10):e330 [FREE Full text] [doi: 10.2196/jmir.8360] [Medline: 28970188]

45. Grunfeld E, Fitzpatrick R, Mant D, Yudkin P, Adewuyi-Dalton R, Stewart J, et al. Comparison of breast cancer patient satisfaction with follow-up in primary care versus specialist care: Results from a randomized controlled trial. Br J Gen Pract 1999 Sep;49(446):705-710 [FREE Full text] [Medline: 10756611]

46. Brennan M, Butow P, Spillane A, Boyle F. Survivorship care after breast cancer: Follow-up practices of Australian health professionals and attitudes to a survivorship care plan. Asia Pac J Clin Oncol 2010 Jun;6(2):116-125. [doi: 10.1111/j.1743-7563.2010.01286.x] [Medline: 20565424] 


\section{Abbreviations}

HL7: Health Level Seven

ISLHD: Illawarra Shoalhaven Local Health District

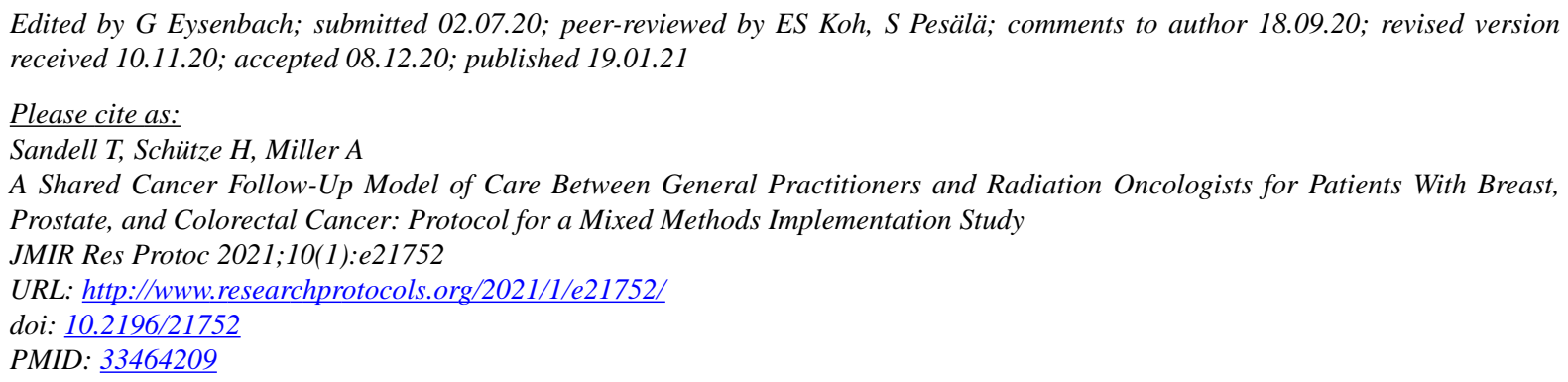

CTiffany Sandell, Heike Schütze, Andrew Miller. Originally published in JMIR Research Protocols (http://www.researchprotocols.org), 19.01.2021. This is an open-access article distributed under the terms of the Creative Commons Attribution License (https://creativecommons.org/licenses/by/4.0/), which permits unrestricted use, distribution, and reproduction in any medium, provided the original work, first published in JMIR Research Protocols, is properly cited. The complete bibliographic information, a link to the original publication on http://www.researchprotocols.org, as well as this copyright and license information must be included. 